\title{
Des oscillations non linéaires des eaux portuaires
}

\section{Non-linear oscillations of harbour waters}

\author{
PAR P. GAILLARD \\ ASSISTANT A LA FACULTÉ DES SGreNCES JE GRENOBLE
}

Communication présentée à la Société Hydrotechnique de France le 18 juin 1959

\begin{abstract}
L'étude théorique des oscillations des eaux dans un bassin rectangulaire de profondeur constante soumis $\dot{a}$ travers une passe d̀ l'action d'une houle complexe est ici poussée all second ordre d'approximation par rapport anx amplitudes. Au premier ordre, l'étude développée par Achynt Apté est reprise de manière plus rigouleuse. Au second ordre apparaissent des oscillations de longue période.

On peut, en particulier, interpréter à l'aide des effets non linéaires l'apparition des seiches, nuisibles à la stabilité des navires amarrés, excitées dans le port par une houle de période conte mais variable venant du large.
\end{abstract}

\begin{abstract}
The author extends the theoretical study of water oscillations in a rectangular basin of constant depth, subjected to the action of a complex swell through an opening in the basin, to the second order of approximation for amplitudes. The second order treatment due to Achyut Apte is developed more rigorously. The second order treatment brings out long period oscillations.

Using non-linear effects it is possible to interpret the creation of seiches, which endanger the stability of moored ships and which are excited in harbours by short but variable period swells coming from the open sea.
\end{abstract}

Le sujet de la présente étude a été suggéré par M. J. Larras; il s'agit d'interpréter théoriquement un phénomène souvent observé dans les bassins portuaires, que nous décrirons après en avoir défini les effets nuisibles.

Des mesures faites sur modèle réduit, des théories approchées de Le Méhauté et de Sommet et des constatations de la pratique courante d'ingénieur, il résulte que la sécurité des navires ancrés ou amarrés dans un bassin portuaire agité par une seiche, dépend de la période de celle-ci. La rupture des amarres ne peut avoir lieu que pour les seiches de période comprise entre 30 et 180 secondes suivant le tonnage du navire, les propriétés mécaniques et le mou des aussières; mais alors les accidents se produisent même avec des seiches de faible amplitude, de l'ordre de 2 ou 3 décimètres. On voit donc combien les oscillations des eaux portuaires de longue période peuvent être dangereuses pour les navires abrités dans un bassin. Il y a ainsi un inté- rêt majeur à étudier les conditions de génération des seiches de longue période.

Or une oscillation périodique des eaux des bassins est toujours induite par la houle venant du large. Dans le cadre de la théorie linéaire (cf. les thèses de Mc Nown [1] et d'Apté [2]), les périodes des deux phénomènes sont nécessairement les mêmes. Il en résulterait qu'un train de vagues incidentes courtes venant de la haute mer ne pourrait exciter dans le port que des oscillations de brève période. Or l'expérience montre qu'il n'en est pas toujour's ainsi. Dans l'exemple cité par M. Larras, la houle de $10 \mathrm{~s}$ de période provoquait dans le port d'Alger une seiche secondaire de période voisine de $100 \mathrm{~s}$ dont l'approximation linéaire est impuissante à interpréter la naissance.

C'est pourquoi MM. J. Kravtchenko et I. Santon nous ont proposé de mettre au point une théorie du second ordre qui permet d'interpréter le phénomène de la naissance des seiches 
agitant le bassin portuaire selon un schéma non linéaire, inspiré des travaux de F. Biesel [3] [4] sur la houle irrégulière plane.

Imaginons qu'au premier ordre d'approximation, la houle incidente soit, non pas une onde monopériodique, mais comprenne des oscillations irrégulières présentant un certain spectre discontinu de fréquences. Diverses études théoriques des oscillations non linéaires - le calcul de Biesel et ses extensions ultérieures, dues à Kravtchenko, Daubert [5], Normandin [6], Gaillard [7], etc., -- ont mis en évidence la présence, aux approximations d'ordre supérieur au premier, d'ondes d'accompagnement traduisant l'interaction entre les diverses composantes $\mathrm{du}$ spectre linéaire. Ainsi, des composantes linéaires de l'agitation de périodes $T_{1}$ et $T_{2}$ voisines admettent au second ordre des ondes d'interaction de périodes:

$$
\begin{gathered}
\mathrm{T}^{\prime}=\frac{\mathrm{T}_{1} \mathrm{~T}_{2}}{\mathrm{~T}_{1}+\mathrm{T}_{2}}\left(\mathrm{~T}^{\prime}<\mathrm{T}_{2}<\mathrm{T}_{1}\right) \\
\mathrm{T}^{\prime \prime}=\frac{\mathrm{T}_{1} \mathrm{~T}_{2}}{\mathrm{~T}_{1}-{ }^{\mathrm{T}} \mathrm{T}_{2}}\left(\mathrm{~T}^{\prime \prime}>\mathrm{T}_{1}>\mathrm{T}_{2}\right)
\end{gathered}
$$

el

Soulignons que, dans une série de publicalions importantes [8], N.N. Moisseev a développé les théories analogues à la nôtre dans le cas des bassins limités de forme quelconque. Cet auteur ne cherche aucun résultat numérique, mais fonde une théorie générale : il développe le mécanisme du calcul des approximations d'ordre quelconque, montre que celles-ci peuvent être évaluées par un algorithme régulier et indique les corrections non linéaires pour la formule des fréquences.

Au contraire, notre but est de donner des formules explicites relatives à un cas particulier et susceptibles d'applications numériques : cas d'un bassin rectangulaire de profondeur constante, ouvert sur un canal de section constante, ce dernier étant terminé par un batteur à houle dont le mouvement admet un spectre de fréquences discontinu. M. Larras a signalè qu'un tel ouvrage schématise bien un port doté d'un avant-port.

Notre mémoire comprend deux parties. Nous exposerons d'abord la solution formelle la plus générale du problème du premier ordre, explicitée par Takano, et qui généralise les résullals bien connus de M. Apté. Nous avons légitimé cette solution en étudiant les questions de convergence.

La deuxième partie fait connaître les calculs de l'approximation du second ordre; une première variante, due à Takano, et poussée jusqu'aux applications numériques, donne la solution relative à des données frontières a priori choisies dans la passe d'une manière propre à représenter de façon approchée un phénomène réel. Ensuite nous indiquons le calcul formel d'une solution correspondant aux conditions réelles d'une expérience. Mais ici l'application numérique reste à faire.

L'étude théorique et expérimentale des oscillations stationnaires, irrotationnelles, monopériodiques, dans le mème type d'ouvrage, avait été entreprise avec succès au premier ordre par A. Apté. Cet auteur ramène le problème tridimensionnel à un problème plan en recherchant le potentiel des vitesses inconnu $\Phi_{1}(x y z t)$ sous la forme particulière, classique en canal indéfini de profondeur constante :

$$
\Phi_{1}=\sin 2 \pi\left(t /{ }^{\prime} \mathrm{T}\right) \frac{\operatorname{ch} \omega_{0}(z+h)}{\operatorname{ch} \omega_{0} h} \mathrm{~F}(x, y)
$$

le nombre d'onde $\omega_{0}$ étant solution de l'équation d'Airy :

$$
(2 \pi / \mathrm{T})^{2}=g \omega_{0} \text { th } \omega_{0} h
$$

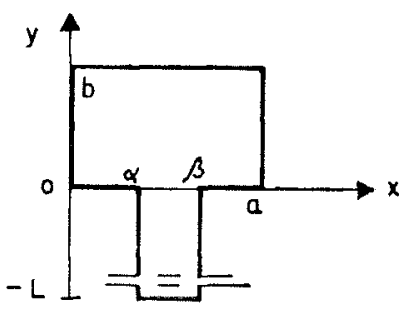

Les axes Oxyz sont orientés comme suit : $\mathrm{O} z$ vertical, ascendant : $O x, O y$, horizontaux dans le plan de la surface libre au repos, l'origine étant située sur l'une des arêtes communes à deux parois du bassin. Le problème mixte, posé relativement à l'équation :

$\frac{\partial^{2} \Phi_{1}}{\partial x^{2}}+\frac{\partial^{2} \Phi_{1}}{\partial y^{2}}+\frac{\partial^{2} \Phi_{1}}{\partial z^{2}}=0$ avec les conditions aux limites:

$\frac{\partial^{2} \Phi_{1}}{\partial t^{2}}+g \frac{\partial \Phi_{1}}{\partial z}=0 \quad$ à la surface libre $(z=0)$

$\frac{\partial \Phi_{1}}{\partial n}=\left\{\begin{array}{c}0 \\ -\partial \eta / d t\end{array}\right.$

le long des parois et sur le fond au droit du batteur $(y=-\mathbf{L})$

où $n$ et $\eta_{i}(x z t)=f(x, z) \cos$ vt désignent respectivement la normale positive dirigée vers l'extérieur du domaine et l'équation au premier ordre de la frontière constituée par la surface du batteur en mouvement — était ramené au problème de Neumann, posé relativement à l'équation de la membrane : 
$\Delta \mathbf{F}+\omega_{1}, \mathbf{F}=0$ avec les conditions aux limiles :

$\frac{d \mathbf{F}}{d n}=\left\{\begin{array}{cl}0 & \text { aux parois et sur le fond } \\ -f(x) \text { au droit du batteur }\end{array}\right.$

où :

$$
\eta(x, z, t)=f_{0}(x) \frac{\operatorname{ch} \omega_{0}(z+h)}{\operatorname{ch} \omega_{0} h} \cos v t
$$

Cetle hypothèse tient compte de l'éventuelle courbure du batteur : elle permet par conséquent de traiter le cas des houles obliques dans le canal [cf. thèse d'Apté, p. 81, formule $(7,7)]$, pour peu que leur demi-longueur d'onde soit inférieure à la largeur du canal; cette hypothèse offre l'inconvénient d'astreindre le batteur à avoir une forme épousant le profil des déplacements des particules sous l'effet de la houle en canal indéfini. On sait qu'une telle condition ne peut être réalisée par un générateur à houle doté d'un volet rigide. Or la théorie de la génération linéaire de la houle plane, due à Havelock-Biesel [9], montre que, même pour un batteur plan, il existe dans le voisinage de son volet des oscillations d'une forme différente de (1); les potentiels correspondants sont des fonctions sinusoïdales de la profondeur et tendent exponentiellement vers zéro lorsqu'on s'éloigne du batteur.

Autrement dit, la forme (1) imposée a priori a $\Phi_{1}$, se trouve ètre trop particulière; elle ne frut vérifier loutes les conditions aux limites au premier ordre, encore qu'elle donne une bonne approximation des faits courants. C'est pourquoi nous avons cru à propos de reprendre la question en prenant en compte toutes les oscillations linéaires de période $T$ dont le bassin peut être le siège. D'autre part, en rue de comparer nos résultats aux précédents, nous avons conservé l'hypothèse de non-planéité du batteur, bien qu'elle ne soil d'aucune utilité pratique pour nous. Le cas général est ainsi, et grâce à la linéarité des équations qui permet de développer $r_{1}(x, z, t)$ et $\Phi_{1}(x, y, z, t)$ en série de Fourier par rapport au temps, ramené à la recherche d'une solution :

$$
\Phi_{1}(x, y, z, t)=\varphi_{1}(x, y, z) \sin v t
$$

du système (3) pour un mouvement donné du batteur.

Pour résoudre ce problème, nous utilisons unc méthode de calcul approchée appliquée par Apté et dont nous rappelons le principe. On décompose le domaine port-canal en deux parallélépipèdes rectangles à l'aide du plan $y=0$; on pose :

$$
\begin{aligned}
& \varphi_{1}=\varphi_{p r} \text { dans le port } y \geqslant 0 \text { (domaine } \mathrm{D}_{p} \text { ) } \\
& \varphi_{1}=\vartheta_{c 1} \text { dans le canal } y \leqslant 0 \text { (domaine } \mathrm{D}_{e} \text { ) }
\end{aligned}
$$

On introduit une nouvelle inconnue $g_{1}(x, z)$ :

$$
\begin{array}{cc}
\frac{\partial \varphi_{p 1}}{\partial y} & =\frac{\partial \varphi_{i 1}}{\partial y}=g_{1}(x, z) \\
y=0 ; \quad & \alpha<x<\beta \quad-h \leqslant z \leqslant 0
\end{array}
$$

$g(x, z)$ étant supposée connue, $\varphi_{p}$ et $\varphi_{o}$ sont déterminés dans le port et le canal respectivement comme solutions d'un problème mixte de Neumann-Cauchy.

Il suffit alors d'exprimer que ces fonctions sont prolongements analytiques l'une de l'autre; à cet effet, il suffit d'écrire :

$\varphi_{p 1}=\varphi_{a 1}$ pour $\quad y=0 ; \alpha<x<\beta ;-h \leqslant z \leqslant 0$

on verra que cette relation délermine completement $g_{1}(\boldsymbol{x}, \boldsymbol{z})$ formellement, du moins.

La fonction $o_{1}(x, y, z)$, harmonique et régulière dans son domaine de définition (c'est-à-dire finie et continue avec les dérivées des deux premiers ordres finies en chacun de ses points frontières), n'est pas uniforme autour des arêtes verticales communes aux parois du port et du canal. Il est essentiel, pour la validité des raisonnements, de déterminer a priori la nature de cette singularité du potentiel. Si $\varphi_{1}(x, y, z)$ est de la forme (1), la question est traitée dans [10]; on trouve :

$$
\begin{gathered}
\left|\varphi_{1}(x, y, z)-\varphi_{1}(0,0, z)\right| \sim \rho^{2 / 3} \\
g_{1}(x, z)=\left|\frac{\partial \varphi_{1}(x, y, z)}{\partial y}\right|_{y=0} \sim \varphi^{-1 / 3}
\end{gathered}
$$

Nous n'avons pas réussi à étendre en toute rigueur celte conclusion au cas général; quelques raisonnements préliminaires nous autorisent à penser que les relations (11) sont encore valables dans le cas qui nous occupe.

Considérons les suites infinies de valeurs propres $\mathbf{K}_{m n p}$ et des fonctions propres $\psi_{1 m n p}$ $(m, n, p=0,1,2 \ldots)$ :

$$
\Delta \bigcup_{1}+\mathrm{K}^{2} \psi_{1}=0
$$

relalivement au domaine $D_{p}$ et aux conditions frontières :

$\frac{\partial \psi_{1}}{d n}=0$

le long des parois et sur le fond

$g \frac{\partial \downarrow_{1}}{\partial z}-v^{2} \psi_{1}=0$ à la surface libre $(z=0)$ 
On a :

$$
\begin{aligned}
& \mathrm{K}_{1 m n p}= \begin{cases}{\left[\left(\mathrm{m}^{2} / a^{2}\right)+\left(n^{2} / b^{2}\right)\right] \pi^{2}-\omega_{0}{ }^{2},} & p=0 \\
{\left[\left(m^{2} / a^{2}\right)+\left(n^{2} / b^{2}\right)\right] \pi^{2}+\left(\omega_{p^{2}}{ }^{2},\right.} & p \geqslant 1\end{cases} \\
& \downarrow_{1 m n p}=\left\{\begin{array}{l}
\cos m \pi(x / a) \cos n \pi(y / b) \operatorname{ch} \omega_{0}(z+h) \\
\cos m \pi(x / a) \cos n \pi(y / b) \cos \omega_{p}(z+h) ; p \geqslant 1, v^{2}=-g \omega_{p} \operatorname{tg} \omega_{p} h
\end{array}\right.
\end{aligned}
$$

La suite des $\Psi_{1 \text { mnp }}$ étant complète et orthogonale, on sait que toute fonction assez régulière dans $D$ est développable en série de Bessel-Fourier suivant $\psi_{1 m n p}$. Si donc la solution remplit les conditions requises de régularité, on peut la définir au moyen d'un développement du type :

$$
\varphi_{p 1}(x, y, z)=: \sum_{m=0}^{\infty} \sum_{n=0}^{\infty} \sum_{p=0}^{\infty} \mathrm{D}_{1 m n}, \psi_{1 m n p}
$$

dont les coefficients constants $D_{1 m n p}$ sont donnés par :

$$
\mathrm{D}_{1 m n p}=\frac{\iiint_{\supset_{n}} \psi_{1 m n p} \varphi_{p 1} d \tau}{\iiint_{D_{n}} \psi^{2}{ }_{1 m n p} d \tau}
$$

où $d \tau$ est l'élément de volume de $\mathrm{D}_{p}$. Or, les propriétés énoncées de ơp $(x, y, z)$ et de $g_{1}(x, z)$ garantissent - - ce qu'on montre en adaptant à notre cas les raisonnements de Kravtchenko et Apté - que cette représentation (16) de la solution est valable, mais elle est impropre au calcul, par dérivation terme à terme, des dérivées partielles de $\varphi_{p 1}$, ce calcul formel conduisant à des séries divergentes. Ceci tient à ce que nous avons utilisé un développement de Bessel-Fourier pour représenter une fonction harmonique, aucune des fonctions de la suite complète $\Downarrow_{1 \mathrm{mnp}}$ n'étant harmonique et ne vérifiant toutes les conditions aux Jimites (3). Ainsi la détermination de $\varphi_{p 1}$ revient au calcul des coefficients de BesselFourier $D_{1 m n p}$. Les propriétés de régularité de $\vartheta_{n 1}$ et de $g_{1}(x, z)$ permettent d'appliquer la formule de Green :

$$
\begin{gathered}
\iiint_{\mathrm{D}_{p}}\left[\left(\Delta \varphi_{p 1}\right) \psi_{1 m n p}-\varphi_{p 1}\left(\Delta \psi_{1 m n p}\right)\right] d \tau=\iint_{\mathrm{F}_{p}}\left[\frac{d \varphi_{p 1}}{d n} \psi_{1 m n p}-\varphi_{p 1} \frac{d \psi_{1 m n p}}{d n}\right] d r s \\
\mathrm{~K}_{1}{ }^{2}{ }_{m n p} \mathrm{D}_{1 m n p} \iiint_{\mathrm{D}_{p}} \psi_{1 m n p} \partial \tau=-\int_{-n}^{0} d z \int_{\alpha}^{\beta} \psi_{1 m n p} g_{1}(x, z) d x
\end{gathered}
$$

et de représenter $g(x, z)$ au moyen d'un développement du type :

$$
g_{1}(x, z)=\sum_{g_{i, h}=0}^{\infty} \cos m \pi(x / a)\left[\mathrm{U}_{m 0} \operatorname{ch} \omega_{0}(z+h)+\sum_{p=1}^{\infty} \mathrm{U}_{m p} \cos m \pi(x / a) \cos \omega_{p}(z+h)\right]
$$

on en déduit l'expression de $\mathrm{D}_{1 m n p}$ en fonction de $\mathrm{K}_{1 m n p}$ et $\mathrm{U}_{m p}$. Il est possible, en remarquant que :

$$
\sum_{n=0}^{\infty} \mathrm{D}_{1 n n \bar{p}} \cos n \pi(y / b)
$$

est le développement en série de Fourier de fonctions élémentaires, sinusoïdales et hyperboliques, de transformer la série triple (16) en série double. Cette série double est une somme indéfinie de produits de Laplace, c'est-à-dire qu'elle s'identifie à la solution formelle du système (3) (4) du type particulier suivant :

$$
\varphi_{1}(x, y, z)=\sum_{m=0}^{\infty} \sum_{y=0}^{\infty} f_{m}(x) \times g_{m p}(y) \times h_{p}(z)
$$

Le processus de calcul est le même dans le domaine $D_{c}$ ou l'on donne de $f(x, z)$ - cf. formule (4) — une représentation analogue à (20):

$$
f(x, z)=\sum_{m=0}^{\infty} \cos m \pi[(x-\alpha) /(\beta-\alpha)]\left[W_{m 0} \operatorname{ch~}\left(w_{0}(z+h)+\sum_{p=1}^{\infty} W_{m p} \cos \omega_{p}(z+h)\right]\right.
$$


Ial détermination complète des coefficients $\mathrm{U}_{m p}$ et $\mathrm{V}_{m p}$ relatifs respectivement à $\mathrm{D}_{p}$ et à $\mathrm{D}_{v}$, en fonction des $W_{m p}$ s'obtient en portant dans (9) et (10) les représentations, respectivement du type (20) et $(21)$, de $g_{1}(x, z)$ et $\varphi_{1}(x, y, z)$ dans $\mathrm{D}_{p}$ et $\mathrm{D}_{c}$. A chaque indice, $p$ est ainsi associé à un système infini d'équations linéaires par rapport aux inconnues $U_{m p}$ et $V_{m p}$. La résolution de chacun de ces systèmes présente une double difficulté :

a) du point de vue théorique, les théorèmes connus d'existence et d'unicité de la solution s'appliquent à des systèmes infinis dont les coefficients numériques satisfont à certaines conditions de convergence - cf. théorie de Von Koch dont on trouvera un exposé très accessible dans l'ouvrage de F. Riesz [11] —; en première analyse nos systèmes ne semblent pas vérifier ces conditions;

b) du point de vue pratique, en supposant que nos systèmes admettent une solution unique, les diverses méthodes de calcul approché que l'on peut mettre en ouvre conduisent à un résultat dont on ne peut évaluer a priori la précision.

Notre généralisation du calcul d'Apté présente donc encore les mêmes lacunes que celles de cet auteur; nous nous efforçons actuellement de les combler. Les récents travaux de Takano sur le passage de la houle sur un obstacle parallélépipédique se heurtent aux mêmes difficultés.

Ainsi donc, là où Apté utilise une seule fonction propre en $z$ à savoir $\operatorname{ch} \omega_{n}(z+h)$, nous sommes conduits à en introduire une infinité — cf. formule (15). - Or, partant de sa solution particulière, l'auteur précité a obtenu des solutions approchées du problème des seiches portuaires en excellent accord avec les expériences, faites par Marcou et Apté. On peut s'interroger sur l'origine de cette concordance entre l'expérimentation et sa théorie. L'analyse approchée que nous avons faite de nos systèmes infinis permet d'expliquer ce fait a priori. Si, en effet, la longueur du canal est suffisante, les coefficients, dont sont affectées les données $W_{m p}$ dans nos systèmes, ont des valeurs extrêmement petites pour $p \neq 0$, ce qui entraîne pour les inconnues $\mathrm{U}_{m p}$ et $\mathrm{V}_{m p}$ des systèmes relatifs à $p \not \geq 0$ des valeurs pratiquement négligeables vis-à-vis des valeurs des inconnues $U_{m 0}$ et $V_{n \iota 0}$ du système relatif à $p=0$. Dans l'éventualité envisagée, l'approximation d'Apté s'avère donc très satisfaisante.

Dans tout notre exposé, nous supposons que le mouvement dụ batteur résulte de la superposition de $\mathrm{S}$ composantes temporelles de la forme:

$$
\begin{aligned}
\eta_{i}(x, z, t) & =\sum_{j=1}^{\mathrm{s}} r_{i j}(x, z) \cos v_{j} t+\eta_{j}^{*}(x, z) \sin v_{j} t \\
& \left.=\sum_{j=1}^{\mathrm{s}} \cos v_{j} t \sum_{m=0}^{\infty} \cos m \pi[(x-\alpha) /(\beta-\alpha)]\left[\mathrm{W}_{j m 0} \operatorname{ch} \omega_{j 0}(z+h)+\sum_{p=1}^{\infty} \mathrm{W}_{j m p} \cos \omega_{j p}(z+h)\right]\right\} \\
& +\sum_{j=1}^{\mathrm{s}} \sin v_{j} t \sum_{n=0}^{\infty} \cos m \pi[(x-\alpha) /(\beta-\alpha)]\left[\mathrm{W}^{*}{ }_{j m 0} \operatorname{ch} \omega_{j 0}(z+h)+\sum_{j=1}^{\infty} \mathrm{W}_{j m p}^{*} \cos \omega_{j p}(z+h)\right]
\end{aligned}
$$

Ces composantes représentent les harmoniques d'un ou plusieurs mouvements périodiques superposés. Dans le cadre de la théorie linéaire, la solution $\Phi_{1}(x, y, z, t)$ du système d'équations (3), où la donnée (4) est remplacée par (23), est de la forme:

$$
\Phi_{1}(x, y, z, t)==\sum_{j=1}^{S} \varphi_{j}(x, y, z) \sin \nu_{j} t+\varphi_{j}^{*}(x, y, z) \cos v_{j} t
$$

où $\varphi_{j}$ et $\varphi^{*}$ sont déterminés par une représentation du type (21).

Passons maintenant à l'étude non linéaire du phénomène.

Au second ordre d'approximation, le potentiel des vitesses $\Phi(x, y, z, t)$ comporte, outre 
$\Phi_{1}(x, y, z, l)$ représenté par (24), la solution $\Phi_{2}(x, y, z, t)$ d'un problème mixte posé relativement à l'équation :

$$
\begin{aligned}
& \frac{\partial^{2} \Phi_{2}}{\partial x^{2}}+\frac{\partial^{2} \Phi_{2}}{\partial y^{2}}+\frac{\partial^{2} \Phi_{2}}{\partial z^{2}}=0 \text {, avec les conditions aux limites : } \\
& \frac{\partial^{2} \Phi_{2}}{\partial t^{2}}+g \frac{\partial^{2} \Phi_{2}}{\partial z}=-2 \frac{\partial \Phi_{1}}{\partial x} \frac{\partial^{2} \Phi_{1}}{\partial x \partial t}-2 \frac{\partial \Phi_{1}}{\partial y} \frac{\partial^{2} \Phi_{1}}{\partial y \partial t}-\frac{\partial \Phi_{1}}{\partial z} \frac{\partial^{2} \Phi_{1}}{\partial z \partial t} \\
& +1 / g \frac{\partial \Phi_{1}}{\partial t}-\frac{\partial}{\partial z}\left[\frac{\partial^{2} \Phi_{1}}{\partial t^{2}}+g \frac{\partial \Phi_{1}}{\partial z}\right] \text { pour } z=0 \\
& \frac{d \Phi_{2}}{d n}=\left\{\begin{array}{l}
0 \text { le long des parois et sur le fond, } \\
\frac{\partial \Phi_{1}}{\partial x} \frac{\partial \eta}{\partial x}+\frac{\partial \Phi_{1}}{\partial z} \frac{\partial \eta}{\partial z}-\eta \frac{\partial^{2} \Phi_{1}}{\partial y^{2}} \text { pour } y=-\mathrm{L}
\end{array}\right.
\end{aligned}
$$

D'après (24), le second membre de la condition au droit du batteur est de la forme :

$$
\frac{\partial \Phi_{2}}{\partial y}=\sum_{j=1}^{s} \sum_{k=1}^{j}\left\{\begin{array}{l}
\mathrm{E}_{j k}(x, z) \sin \left(v_{j}+v_{k}\right) t+\mathrm{G}_{j k}(x, z) \sin \left(v_{j}-v_{k}\right) t \\
+\mathrm{E}^{*}{ }_{j k}(x, z) \cos \left(v_{j}+v_{k}\right) t+\mathrm{G}_{j k}^{*}(x, z) \cos \left(v_{j}-v_{k}\right)
\end{array}\right\} \text { pour } y=-\mathrm{L}
$$

Il en est de même pour la condition à la surface libre. Cette propriété, ainsi que la linéarité des équations (25) par rapport à $\Phi_{2}$ permet de chercher $\Phi_{2}(x, y, z, t)$ sous la forme:

$$
\Phi_{2}(x, y, z, t)=\sum_{j=1}^{s} \sum_{k=1}^{j}\left\{\begin{array}{r}
\varphi_{2 j k}(x, y, z) \sin \left(v_{j}+v_{k}\right) t+\theta_{2 j k}(x, y, z) \sin \left(v_{j}-v_{k}\right) t \\
+\varphi_{2 j k}^{*}(x, y, z) \cos \left(v_{j}+v_{k}\right) t+\theta_{2 j k}^{*}(x, y, z) \cos \left(v_{j}-v_{k}\right) t
\end{array}\right\}
$$

La méthode d'intégration est identique à celle exposée au premier ordre - on définit les suites de valeurs propres $K_{j k m n p}$ et des fonctions propres $\Psi_{j k m n p}$ de l'équation (12) relativement au domaine $\mathrm{D}_{p}$ et aux conditions frontières (13) où $v_{j}$ est remplacé par $\left(v_{j}+v_{k}\right)$. Les fonctions propres $\psi_{j k m n p}$ sont définies par les relations analogues à $(15)$, où $\omega_{j p}$ est remplacé par $\omega_{j k p}$. Les $\varphi_{2} j_{k}$ sont alors définis au moyen de développements du type :

$$
\varphi_{2 j k}=\sum_{m=0}^{\infty} \sum_{n=0}^{\infty} \sum_{p=0}^{\infty} \mathrm{D}_{i k m n p} \psi_{j k m n \eta}
$$

Onn pose, en outre :

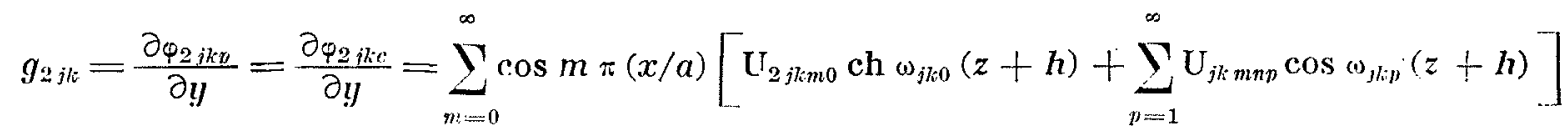

Là encore on arrive à donner de $\varphi_{2 j k}$ une représentation du type (21).

La solution explicitée est formelle : nous n'avons encore pu établir que les fonctions inconnues :

$$
\varphi_{2 j k}, \quad \theta_{2 j k}, \varphi_{2 j k}^{*}, 0_{2 j k}^{*}, g_{2 j k}, e_{2 j k}, g_{2 j k}^{*}, e_{2 j k}^{*}
$$

- $e_{2 j k}$ étant lié à $\theta_{2 j k}$ par une relation analogue à (29) où $R, S$ remplacent $U, V$ - jouissent de propriétés de régularité suffisantes pour admettre les développements que nous avons utili- sés pour les représenter. Par cette méthode, la représentation (27) de la solution est à présent explicitée en fonction des paramètres. Mais il reste à franchir une ultime étape : écrire et résoudre les équations analogues à (9) et (10) qui déterminent les coefficients inconnus $\mathrm{U}_{j k m p}$, $\mathrm{V}_{i k m p}, \mathrm{U}^{*}{ }_{j k m p}, \mathrm{~V}^{*}{ }_{j k, n p}, \mathrm{R}_{j k m p}, \mathrm{~S}_{j k_{m}, p}$, etc., et par suite $\mathrm{D}_{j k m u p}$ en fonction des donnces $\mathrm{W}_{j m p}$ et $\mathrm{W}^{*}{ }^{*}{ }$.

Ici nous présentons une remarque qui nous 
semble digne d'intérèt. Dans la généralité des cas, le calcul approché des oscillations stationnaires a été tenté dans l'une des deux hypothèses : $a$ ) le mouvement est irrotationnel; $b$ ) le mouvement ne présente aucun transport de masse. Les variables d'Euler semblent adaptées à l'exploitation de la première hypothèse, les variables de Miche à celles de la seconde. Dans les cas bidimensionnels étudiés jusqu'ici, il a été constaté que ces deux hypothèses étaient équivalentes au second ordre; l'absence de transport de masse entraîne l'existence du potentiel de vitesse et inversement. Chabert d'Hières a même étendu cette propriété à tous les ordres d'approximation dans le cas du clapotis parfait. Nous avons calculé le rotationnel correspondant à la seconde hypothèse et nous sommes arrivés à la conclusion que voici :

a) Si le mouvement du batteur défini par (23) peut se réduire à la forme suivante :

$$
\eta(x, z, t)=\sum_{j=1}^{\beta} f_{j}(x, z) \cos \left(v_{j} t+\rho_{j}\right)
$$

où les phases $\rho_{j}$ sont des constantes, c'est-à-dire sont indépendantes de $x$ et de $z$, il est alors possible de chercher une solution $\Phi_{1}$ du type :

$$
\Phi_{1}(x, y, z, t)=\sum_{j=1}^{s} \rho_{j}(x, y, z) \sin \left(y_{j} t+c_{j}\right)
$$

Il s'ensuit que les oscillations stationnaires tridimensionnelles sont simultanément irrotationnelles et à transport de masse nul, au second ordre inclusivement près. Le mouvement du batteur-piston et celui du batteur en rotation autour d'un axe horizontal font notamment partie de ce type (30), car il existe une phase de leur mouvement où le volet batteur se trouve contenu dans le plan vertical d'abscisse $y=-\mathrm{L}$.

b) Si le mouvement du batteur présente sous la forme (30) une phase ?; variable avec $x$ el $z$, les trois composantes du vecteur tourbillon se composent d'interactions du second ordre entre les termes constituant $\Phi_{1}$ de même période et de phases différentes, à savoir entre $\varphi_{j}$ et $\stackrel{0}{*}_{j}$. La solution stationnaire sans transport de masse comporte alors un champ tourbillonnaire bien déterminé et la solution irrotationnelle un champ de transport de masse.

Une méthode plus rapide mais, peut-être, moins précise a été utilisée par K. Takano qui a fait les hypothèses suivantes :

a) Au lieu de considérer le cas de lagitation multipériodique engendrée par un batteur à houle, Takano se donne arbitrairement et a priori, comme condition aux limites, le champ des composantes longitudinales de vitesse dans une section droite du canal, sous la forme :

$\frac{\partial \Phi}{\partial y}=\mathrm{G}(x, z, t) \quad$ pour $y=-\mathrm{L}$.

Il fait intervenir cette donnée intégralement dans la théorie linéaire, de sorte que la condition s'écrit au premier et au second ordre :

$\frac{\partial \Phi_{1}}{\partial y}=\mathrm{G}(x, z, t) ; \frac{\partial \Phi_{2}}{\partial y}=0$ pour $y=-\mathrm{L}$

Un choix judicieux de $G(x, z, t)$ permet de représenter convenablement les phénomènes réels.

b) Nous avons déjà souligné que les termes en $\cos \omega_{j p}(z+h)$ semblent négligeables dans le bassin et dans le canal au voisinage de la passe, eu égard à la grande longueur du canal; Takano, à la suite d'Apté, n'en a pas tenu compte.

c) Les termes parasites, mis en évidence par Havelock-Biesel, dans le cas d'un canal semiindéfini, d'un batteur plan, et qui varient en fonction de $y$ comme $e^{-\omega_{j p}(y+L)}$ remplacent, eu égard à la grande longueur du canal par rapport à la profondeur, les termes analogues, qui, dans le cas d'un canal borné, varient comme - sh $\omega_{j p} y$.

K. 'Takano a effectué, au moyen de sa méthode, un calcul numérique approché de l'amplitude, en certains points du bassin, des oscillations de longue période, avec la donnée $\mathrm{G}(x, z, t)$ suivante:

$$
\begin{aligned}
G(x, z, t)=a_{1} \operatorname{ch} \omega_{1,0}(z+h) \sin \nu_{1} t \\
\quad+a_{2} \operatorname{ch} \omega_{2.0}(z+h) \sin \nu_{2} t
\end{aligned}
$$

ò̀ $a_{1}$ et $a_{2}$ désignent respectivement les amplitudes de variation au fond des vitesses de périodes ' $\mathrm{T}_{1}$ et ' $\Gamma_{2}$; et $\omega_{1.0}, \omega_{2,0}$ sont liés aux périodes $\mathrm{T}_{1}$ et $\mathrm{T}_{2}$ par la relation d'Airy (2).

Voici le schéma du calcul. La forme de $\boldsymbol{G}(x, z, t)$ ci-dessus montre qu'au premier ordre près, le port sera agité par deux seiches de périodes $T_{1}$ et $T_{2}$ calculables par Je procédé d'Apté. La méthode indiquée ci-dessus permet de former les équations de deux seiches non linéaires de périodes $\left(T_{1}{ }^{\prime} T_{2}\right) /\left(T_{1}-T_{2}\right)$ et $\left(T_{1} T_{2}\right) /\left(T_{1}+T_{2}\right)$ satisfaisant à toutes les conditions aux limites à l'exception de celles relatives à la passe et aux parois verticales situées dans le plan $y=0$. On superpose alors à ces mouvements du second ordre des seiches linéaires de mêmes périodes, assujetties à vérifier le long des parois verticales du port les conditions aux limites telle que l'agitation résultante de période $\left(T_{1} T_{2}\right) /\left(T_{1}-T_{2}\right)$ et $\left(\mathrm{T}_{1} \mathrm{~T}_{2}\right) /\left(\mathrm{T}_{1}+\mathrm{T}_{2}\right)$ soit tangentielle le long de l'élé- 
ment considéré de frontière (cf. formule 34 ), la superposition de tous ces mouvements de longue période donne une seiche portuaire complexe, satisfaisant à toutes les conditions aux limites imposées au second ordre. Le tableau ci-dessous indique les valeurs $A_{1}, A_{2}$, et $A_{1,2}$, de l'amplitude des oscillations de la surface libre de périodes respectives $T_{1}, T_{2}$ et $\left(T_{1} T_{2}\right) /\left(T_{1}-T_{2}\right)$ dans le cas du bassin du laboratoire :

$a=340 \mathrm{~cm} ; b=300 \mathrm{~cm} ; h=30 \mathrm{~cm} ; \mathrm{A}_{1}, \mathrm{~A}_{2} A_{1,2}$ sont exprimés en $\mathrm{cm}, a_{1}$ et $a_{2}$ en $\mathrm{cm} / \mathrm{s}$.

\begin{tabular}{|c|c|c|c|c|c|c|c|}
\hline \multicolumn{2}{|c|}{ Périodes } & \multicolumn{2}{|c|}{$\begin{array}{l}\text { Position du } \\
\text { point d'ob- } \\
\text { servation }\end{array}$} & \multirow{2}{*}{$\begin{array}{c}\begin{array}{c}\text { Profon- } \\
\text { deur } \\
\text { rela- } \\
\text { tive }\end{array} \\
h / \lambda_{.2}\end{array}$} & \multicolumn{3}{|c|}{ Amplitudes } \\
\hline $\mathrm{T}_{1}$ & ${ }_{\mathrm{s}} \mathrm{T}_{2}$ & $\begin{array}{c}x \\
\mathrm{~cm}\end{array}$ & $\begin{array}{c}y \\
\mathrm{~cm}\end{array}$ & & $\begin{array}{l}\mathrm{A}_{1} \\
\mathrm{~cm}\end{array}$ & $\begin{array}{l}A_{e} \\
\mathrm{~cm}\end{array}$ & $\begin{array}{c}A_{1,2} \\
\mathrm{~cm}\end{array}$ \\
\hline 3,3 & 3,0 & 0 & 300 & 0,06 & $0,1 a_{1}$ & $0,4 \quad a_{.,}$ & $0,003 \quad a_{1} a_{s}$ \\
\hline 3,3 & 3,0 & 0 & 150 & 0,06 & $0,1 a_{1}$ & $0,1 \quad a_{2}$ & $0,005 \quad a_{1} a:$ \\
\hline 2,2 & 2,0 & 0 & 300 & 0,09 & $0,3 a_{1}$ & $0,2 \quad a_{2}$ & $0,002 \quad a_{1} a:$ \\
\hline 1,1 & 1,0 & 0 & 300 & 0,25 & $0,19 a_{1}$ & $0,16 a_{2,2}$ & $0,0004 a_{1} a_{:}$ \\
\hline 2,2 & 2,0 & 0 & 150 & 0,09 & & & $0,002 \quad a_{1} a_{2}$ \\
\hline
\end{tabular}

Les résultats du précédent tableau nécessitent deux remarques concernant la légitimité, l'une du calcul aux faibles profondeurs relatives, l'autre celle de l'hypothèse (34) faite sur $\mathrm{G}(x, z, t)$.

Les périodes retenues pour le calcul numérique sont grandes; il s'ensuit que la profondeur relative $h / \lambda$ est faible. Dans le cas bidimensionnel (houle pure, clapotis parfait) on sait que le rayon de convergence des développements de Poincaré qui donnent la solution sous forme de série de Taylor du petit paramètre $\varepsilon$, à savoir la cambrure :

$$
\Phi(x, y, z, t)=\sum_{n=1}^{\infty} \varepsilon^{n} \Phi_{n}(x, y, z, t)
$$

lend vers zéro avec la profondeur relative. Dans le cas tridimensionnel et dans la zone portuaire où Takano a calculé sa solution, les séries du type (21) qui définissent $\Phi_{1}, \Phi_{2}$ ainsi que $A_{1}, A_{2}$ et $A_{1,2}$ convergent rapidement aux faibles profondeurs relatives, mais on ne sait si les séries du type (35) définissant $\Phi$ et la dénivellation de la surface libre convergent sous les hypothèses utilisées pour le calcul numérique. Cette difficulté, bien connue, se traduit, comme l'ont souligné notamment Biesel pour la houle irrégulière au second ordre [3] et Chabert d'Hières pour le clapotis parfait au troisième ordre [13], par une croissance très rapide de l'amplitude des oscillations d'ordre supérieur au premier aux trìs faibles profondeurs relatives (la rapidité de cette croissance croît avec la cambrure). Si on suppose que $A_{1}=1 \mathrm{~cm}, A_{2}=1 \mathrm{~cm}$, on observe au point $(x=0, y=300 \mathrm{~cm})$ les valeurs suivantes de $A_{1,2}$ pour $h / \lambda_{2}=0,25 ; 0,09 ; 0,06$ respectivement: $0,13 \mathrm{~mm}, 0,33 \mathrm{~mm}, 0,75 \mathrm{~mm}$. (Si nous avions fait un calcul à cambrure constante, la croissance aurait été beancoup plus rapide.)

En ce qui concerne l'hypothèse (34) relative à $\mathrm{G}(x, z, t)$, il convient de souligner qu'elle est très particulière et qu'il faut s'attendre généralement à ce que figurent dans $g(x, z, t)$ non seulement les périodes $T_{j}$ des houles linéaires excitatrices, mais celles de leurs interactions non linéaires, à savoir $\left(\mathrm{T}_{j} \mathrm{~T}_{k}\right) /\left(\mathrm{T}_{j}-\mathrm{T}_{k}\right)$ et $\left(\mathrm{T}_{j} \mathrm{~T}_{k}\right) /\left(\mathrm{T}_{j}+\mathrm{T}_{k}\right)$ au second ordre.

La présence de telles périodes d'oscillation dans l'expression de $\mathrm{G}(x, z, t)$ se traduirait par l'apparition dans le port de seiches linéaires de périodes $\left(\mathrm{T}_{1} \mathrm{~T}_{2}\right) /\left(\mathrm{T}_{1}-\mathrm{T}_{2}\right)$ et $\left(\mathrm{T}_{1} \mathrm{~T}_{2}\right) /\left(\mathrm{T}_{1}+\mathrm{T}_{2}\right)$ calculables par le procédé d'Aplé.

A titre de comparaison, nous donnons l'amplitude des oscillations non linéaires planes en canal indéfini de profondeur constante, dont le calcul numérique a été effectué par K. Takano.

\begin{tabular}{|c|c|c|c|c|}
\hline $\begin{array}{l}\mathrm{l}_{1} \\
\mathrm{~s}\end{array}$ & $\begin{array}{l}\mathrm{T}_{2} \\
\mathrm{~s}\end{array}$ & $\begin{array}{l}A_{1} \\
\mathrm{~cm}\end{array}$ & $\begin{array}{l}\mathrm{A}_{2} \\
\mathrm{~cm}\end{array}$ & $\begin{array}{c}A_{1,2} \\
\mathrm{~cm}\end{array}$ \\
\hline 3,3 & 3,0 & $1,94 a_{1}$ & $2,14 a_{2}$ & $0,25 a_{1} a_{2}$ \\
\hline 2,2 & 2,0 & $2,9 \quad a_{1}$ & $3,2 \quad a_{2}$ & $0,95 \quad a_{1} a_{2}$ \\
\hline
\end{tabular}

On voit donc que la théorie qui nous occupe est très complexe. Elle rend compte de l'apparition de seiches de longue période sous l'action de la houle complexe de courte période, mais elle est loin d'être achevée; nous n'en présentons que des aspects partiels.

Au point de vue expérimental, on notera que notre modèle est adapté à produire des oscillations de courte période de l'ordre de $1 \mathrm{~s}$. Or l'amplitude de la seiche de Iongue période correspondante est faible et le phénomène sera difficile à déceler. Le contrôle expérimental de nos prévisions théoriques sera donc délicat; il reste à faire. 


\section{BIBLIOGRAPHIE}

[1] McNown. "Sur l'entretien des oscillations des eaux portuaires sous l'action de la haute mer », 1951. Publications Scientifiques et Techniques du Ministère de l'Air, $\mathrm{n}^{\circ} 278$.

[2] APrÉ (A.). «Recherches théoriques et expérimentales sur le mouvement des liquides pesants avec surface libre », 1957. Publications Scientifiques et Techniques du Ministère de l'Air, $\mathrm{n}^{\circ} 333$.

[3] Biesel ( $F$.). " Equations générales de la houle irrégulière. 》La Houille Blanche, mai-juin 1952.

[4] Carry (C.). « Clapotis partiel 》. La Houille Blanche, aoùt-sept. 1953.

[5] Daunerr (A.). «Théorie approchée de la houle pure et de la houle complexe $»$, à paraître dans les Publications Scientifiques et Techniques du Ministère de l'Air.

«Houle complexe au $3^{\circ}$ ordre d'approximation 》 (S.H.F.). La Houille Blanche, juillet 1958, p. 358365.

[6] Normandin (M.). «Sur la théorie du second ordre des phénomènes parasites dans un canal à houle $»$. C.R.A.S. du 25-11-57, t. 245, p. 1880-1882.
[7] Gaillard (P.). Thèse à paraître.

[8] Moisseev (N. N.). "Sur la théorie des oscillations non linéaires des liquides de masse bornée. 》 Prikladnaia Mathematika i Mechanika, t. XXII, fasc $V, 1958$, p. 612-621 (en russe).

[9] Havelock (T. H.). \& Forced Surface Waves on Water. 》Philosophical Magazine, série 7, XVIII, 1929 , p. 569.

BIESEL (F.). « Etude théorique d'un certain type d'appareil à houle. » La Houille Blanche, 1951, p. 975 .

Santon ,(L.). Cours de $3^{\text {e }}$ eycle, Hydrodynamique supérieure, Faculté des Sciences de Grenoble.

[10] Kravtchenko (J.) et Apté (A.). \& Note sur la méthode d'intėgration de Fourier des équations de la physique mathématique. $\gg$ Annales de l'Institut Fourier, t. VII, 1957, p. 329-358.

[11] Riesz (F.). "Systèmes d'équations linéaires à une infinité d'inconnues. 》 Gauthier-Villars, 1913.

[12] Chabert n'Hières (G.). «Sur l'existence d'un potentiel des vitesses pour un clapotis parfait. 》 C.R.A.S. du 24-3-58, t. 246, pp. 1803-1806.

[13] Chabert D'HiÈres (G.). Thèse à paraitre.
M. le Président remercie M. Gaillard et regrette qu'en raison de l'horaire tendu, il n'ait pas pu aborder l'exposé de belles recherches personnelles qu'il a faites pour légitimer en toute rigueur les solutions dites formelles. M. le Président ajoute que ce travaill, consacré à l'exposé des résultats de MM. Gailland et Takano est, à sa connaissance, la première étude aussi complète des oscillations bidimensionnelles au second ordre des liquides pesants avec surface libre.

M. Larras remercie M. Gaillard de la réponse remarquable qu'il vient d'apporter à la question qu'il avait posée jadis à M. Kravtchenko : les houles induites type Biesel-Kravtchenko peuvent-elles provoquer des seiches dans les ports (seiches de très longues périodes) au même titre que les houles simples monopériodiques seules considérées jusqu'ici (périodes moyennes ou faibles).

Enhardi par les succès de M. Gallland, M. Lanras suggère deux nouvelles études en prolongement de la précédente :

- Attaque directe du problème à partir des houles simples qui lenr donnent naissance;

- Problème des faibles profondeurs relatives, comme on en rencontre dans la plupart des ports.

M. le Président rappelle ensuite que le calcul de l'oscillation au second ordre a été entrepris par
M. TAKano en admettant a priori que la dérivée normale du potentiel du second ordre est nulle dans la section d'entrée; M. Takano soutient que cette condition, adoptée pour la commodité du calcul numérique, n'est pas plus arbitraire que d'autres du même ordre.

M. le Président demande alors à M. Takano de dire les raisons qui militent en faveur de cette thèse. M. TAKANo répond en ces termes :

«M. Kravtchenko et moi ne sommes pas d'accord quant à l'importance à attribuer aux conditions le long du batteur. Au $2^{\circ}$ ordre, j'ai négligé les interactions entre le liquide et le volet du batteur et ai admis que les vitesses horizontales, à cet ordre d'approximation, sonl nulles. Je considère que la prise en compte de conditions plus fines exigerait la prise en compte des facteurs de même ordre, tels que la viscosité, la couche limite, le défaut d'étanchéité entre le volet du batteur et les parois du canal. Les approximations successives, au surplus, convergent mal dans le voisinage du batteur; je pense diès lors que la condition que j'ai adoptée n'est ni meilleure ni pire que des conditions plus complexes. »

M. le Président remercie M. le Professeur Takano de la précieuse collaboration qu'il a apportée depuis deux ans au Laboratoire de Mécanique des Fluides de Grenoble.

M. le Président remercie également l'auditoire et lève la séance. 